\title{
Effect of Ethylene Glycol on Structure and Carrier Transport in Highly Conductive Poly(3,4-ethylenedioxythiophene)/poly(4-styrenesulfonate)
}

\author{
Toshiyuki Murakami, Yusaku Mori* and Hidenori Okuzaki*
}

\author{
Nippon Chemi Con Co., 185-1 Marunouchi, Yabuki, Nishishirakawa 969-0235, Japan \\ Fax: 81-248-42-5196, e-mail: toshi@ nippon.chemi-con.co.jp \\ *University of Yamanashi, 4-4-37 Takeda, Kofu 400-8511, Japan \\ Fax: 81-55-220-8554, e-mail: okuzaki@yamanashi.ac.jp
}

\begin{abstract}
Highly conductive films made of poly(3,4-ethylenedioxythiophene) doped with poly(4-styrenesulfonate) (PEDOT/PSS) were prepared by casting the PEDOT/PSS aqueous dispersion of colloidal particles containing ethylene glycol (EG). By addition of 3\% of EG, the electrical conductivity was significantly increased from 3 to $430 \mathrm{~S} / \mathrm{cm}$. The EG was crucially important for (i) removal of the insulating PSS from the surface of the PEDOT/PSS particles, (ii) crystallization of PEDOT molecules, and (iii) aggregation of the particles, which improved both intra- and inter-particle transfer of charge carriers. A further increase of the EG concentration, however, decreased the conductivity, which was due to the morphological inhomogeneity by the phase segregation.
\end{abstract}

Key words: PEDOT/PSS, Conducting polymer, Ethylene glycol, XPS, XRD

\section{INTRODUCTION}

Poly(3,4-ethylenedioxythiophene) doped with poly(4-styrenesulfonate) (PEDOT/PSS), one of the most successful conducting polymers commercially available in the form of aqueous dispersion as colloidal particles, has superior mechanical properties, thermal stability, and high conductivity, which provides potential applications to electrical and optical devices such as transparent electrodes for touch panels and flexible displays [1,2], capacitors [3], microfibers [4-6], Shottky diodes [7], field-effect transistors [8-10], and actuators [11-13]. The resistance of the conductive polymer is, however, too high to fabricate a large-area electronic circuit due to the low electrical conductivity of the commercially available pristine PEDOT/PSS [4,13]. Previously, we have reported that electrical conductivity of the PEDOT/PSS significantly increased by two orders of magnitude by addition of certain amount of ethylene glycol [14]. A similar tendency is observed for other organic solvents such as dimethyl sulfoxide [15], glycerol, polyethylene glycol [16], and sorbitol [17].

Herein, we have dealt with a highly conductive PEDOT/PSS and effect of ethylene glycol on structure and carrier transport properties have been investigated by means of X-ray photoelectron spectroscopy, X-ray diffraction, atomic force microscopy, and temperature dependence of electrical conductivity.

\section{EXPERIMENTAL}

2.1 Materials

Highly conductive grade of PEDOT/PSS (Clevios PH500, H. C. Starck) was commercially available in the form of aqueous dispersion as colloidal particles. The monomer, 3,4-ethylenedioxythiophene was polymerized by chemical oxidation in the presence of poly(4-styrenesulfonic acid) acting as chemical dopant and stabilizing the dispersion in water due to a net negative charge, where the weight ratio between PEDOT and PSS and the doping ratio to be $1: 2.5$ and 0.33 , respectively (Fig.1) [18].<smiles>CC(C)CC(CC(CC(CC(CC(CC(C)(C)C)c1ccc(S(=O)(=O)O)cc1)c1ccc(S(=O)(=O)O)cc1)c1ccc(S(=O)(=O)O)cc1)c1ccc(S(=O)(=O)O)cc1)c1ccc(S(=O)(=O)O)cc1</smiles>

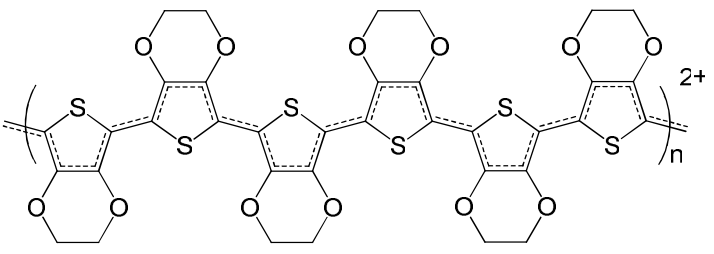

Fig. 1 Chemical structure of PEDOT/PSS.

\subsection{Preparation of films}

Free-standing films were prepared by casting the PEDOT/PSS dispersion containing different amounts of ethylene glycol (EG) in a Teflon dish and allowing it to solidify by evaporation of solvent at $60^{\circ} \mathrm{C}$ for $6 \mathrm{~h}$ and subsequent annealing at $160{ }^{\circ} \mathrm{C}$ for $1 \mathrm{~h}$ in vacuum to remove the $\mathrm{EG}$ completely from the film. 


\subsection{Measurements}

The electrical conductivity of the PEDOT/PSS film was measured by a normal four-probe method with a Lorester (MCP-T610, Dia Instruments). The X-ray photoelectron spectroscopy (XPS) measurements were carried out with an AlK $\alpha$ line (Quantera SXM, Physical Electronics) on the thin films spin-coated on $\mathrm{Si}$ substrates. The X-ray diffraction (XRD) patterns of the free-standing PEDOT/PSS films were measured using an imaging plate (R-AXIS DS3C, Rigaku) at $40 \mathrm{kV}$ and $30 \mathrm{~mA}$ with an exposure time of $1 \mathrm{~h}$. Atomic force microscopic (AFM) measurements were carried out with a scanning probe microscope (SPM-9600, Shimadzu) equipped with a conductive probe. The height images were measured by a tapping mode and current images were measured by a contact mode where a bias of $0.5 \mathrm{~V}$ was applied between the $\mathrm{n}$-doped Si substrate and the conductive probe. Temperature dependence of electrical conductivity was measured by a four-probe technique from $8 \mathrm{~K}$ to $300 \mathrm{~K}$ at a constant heating rate of $1 \mathrm{~K} / \mathrm{min}$ using a cryostat (PS22, Nagase).

\section{RESULTS AND DISCUSSION}

\subsection{Electrical conductivity}

Fig.2 shows dependence of electrical conductivity of the PEDOT/PSS films on EG concentration measured by a normal four-probe method at room temperature. It is found that the conductivity significantly increases by two orders of magnitude from 3 to $430 \mathrm{~S} / \mathrm{cm}$ at EG concentration of $3 \%$ (EG3), which is also higher than that of the Clevios P AG films previously reported by us [11]. A further increase of the EG concentration, however, results in a decrease of the conductivity to $231 \mathrm{~S} / \mathrm{cm}$ at EG concentration of $20 \%$ (EG20) similarly to thin films spin-coated on plastic substrates [14].

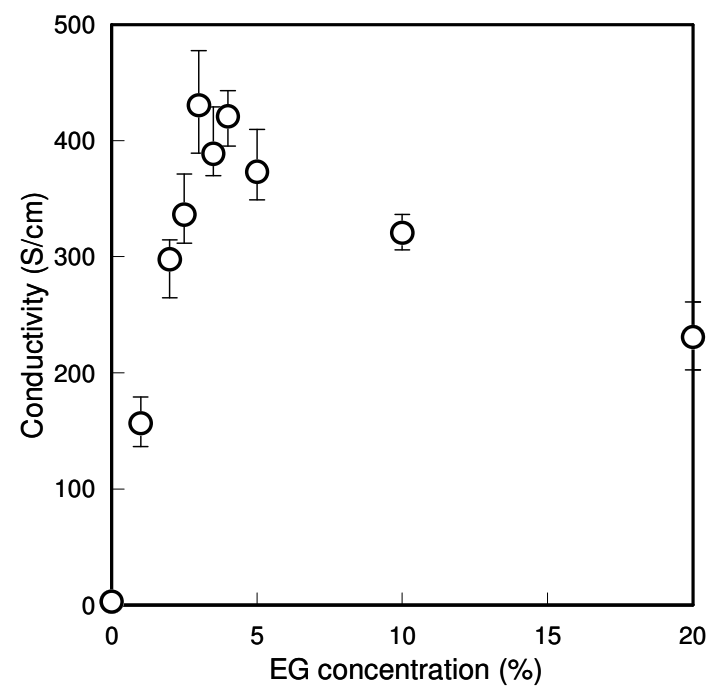

Fig.2 Dependence of electrical conductivity of PEDOT/PSS films on EG concentration measured by a normal four-probe method at room temperature.
3.2 X-ray photoelectron spectroscopy (XPS)

To elucidate the effect of EG on PEDOT to PSS ratio at the surface of the colloidal particles, we performed XPS measurement for the pristine and EG3 films. As demonstrated in Fig.3, the XPS spectra showed well-signed peaks at around 169 and $165 \mathrm{eV}$ due to $S_{2 p}$ of PSS and PEDOT moieties, respectively $[5,19]$. The peak due to the PEDOT moiety significantly increased by addition of EG, where the PEDOT to PSS ratio, calculated using the peak area of $S_{2 p}$ spectra, increased from 0.53 (pristine) to 0.62 (EG3). The result suggested that excess PSS was washed away from the surface region of the PEDOT/PSS particles in the presence of EG, being consistent with the results reported by Jönsson et al. [20]. They found that phase segregation occurred in PEDOT/PSS system and PSS was predominant in the surface region, where the layer of excess PSS surrounding the PEDOT/PSS grains was estimated to be about $37 \AA$ using XPS and UPS [20]. The increase of conductivity is associated with the thinning of the insulating PSS shell surrounding the PEDOT/PSS particles, which improves the contact between the conductive particles that enhances the transport of charge carriers $[5,14]$.

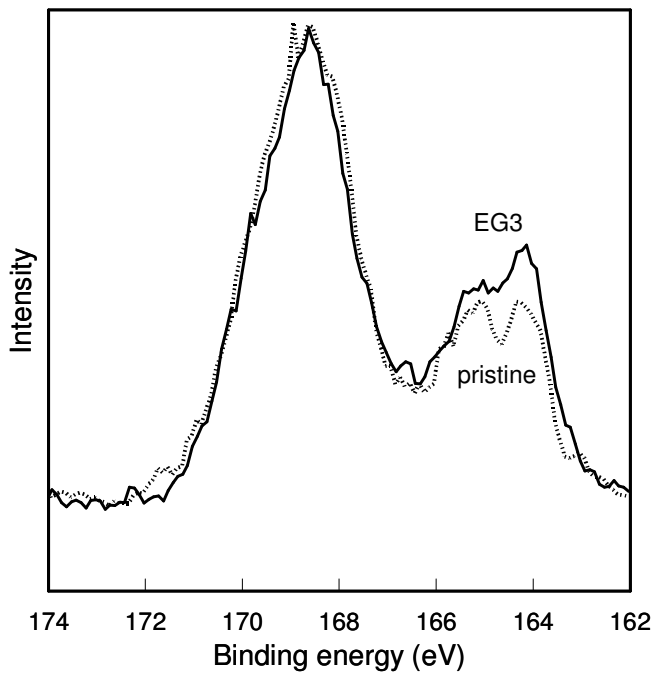

Fig.3 XPS $\left(S_{2 p}\right)$ spectra of pristine (broken line) and EG3 (solid line) films.

\subsection{Crystallinity}

The XRD patterns of various PEDOT/PSS films with different EG concentrations are demonstrated in Fig.4. The pristine film showed halo, indicative of amorphous without higher-order structure of polymer chains. On the other hand, EG3 and EG20 films showed Debye-Scherrer rings at $2 \theta=26^{\circ}(d=$ $3.4 \AA$ ) assigned to (020) plane of an orthorhombic unit cell of PEDOT [21]. This clearly indicates that PEDOT molecules in amorphous state partially change into crystalline state during the film formation. It is considered that the EG, polar organic solvent with higher dielectric constant, reduces the electrostatic interaction between positively charged PEDOT and negatively charged 
PSS in terms of the screening effect, which may enhance the interaction between PEDOT molecules favorable to form a crystalline structure [5].

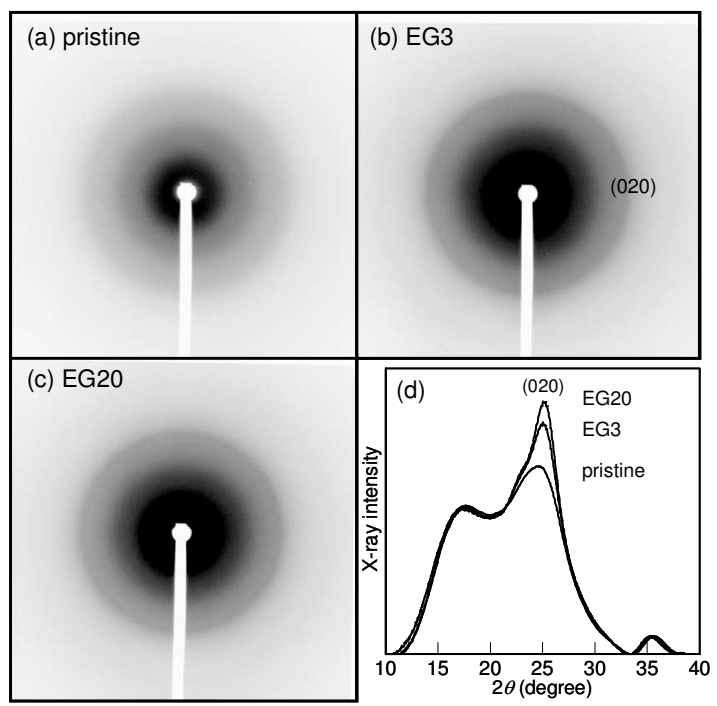

Fig.4 XRD patterns of (a) pristine, (b) EG3, (c) EG20 films measured using an imaging plate and (d) XRD profiles for various films.

\subsection{Surface morphology}

Fig. 5 shows the changes in morphology of the PEDOT/PSS films by addition of EG. It is found that numerous particles with diameters of several tens $\mathrm{nm}$ are densely packed forming the pristine and EG3 films with the same surface roughness $\left(R_{\mathrm{a}}\right)$ of $0.8 \mathrm{~nm}$. On the other hand, for the EG20 film, larger grains loosely aggregate involving structural defects such as holes and cracks where the $R_{\mathrm{a}}$ increases to $1.6 \mathrm{~nm}$. Crispin et al. observed elongated islands due to phase segregation by addition of diethylene glycol (DEG) in AFM images [22]. They also concluded that the addition of DEG reduced excess PSS on the PEDOT/PSS particles by phase segregation. The current images, reflecting local conductivity in the thickness direction, clearly showed that the highly conductive domains (bright area) randomly and loosely distributed in the less conductive matrices (dark area) in the film. It is noted that the highly conductive domains significantly increase in size and in number with increasing the EG concentration.

\subsection{Carrier transport properties}

In order to clarify the effect of EG on carrier transport properties in more detail, temperature dependence of conductivity was measured and the results are shown in Fig.6. It is found that the conductivity of the pristine, EG3, and EG20 films gradually increases from $8 \mathrm{~K}$ to $300 \mathrm{~K}$, indicative of semiconducting characteristics in the experimental temperature range.

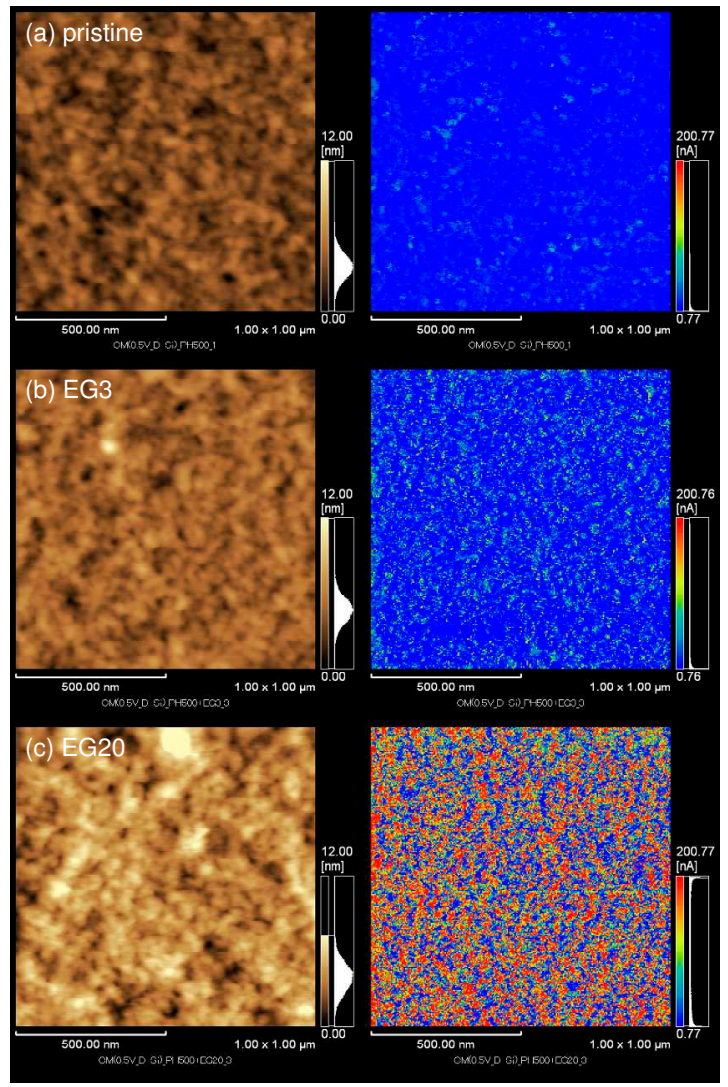

Fig.5 AFM images (left) and current images measured under a bias voltage of $0.5 \mathrm{~V}$ (right) for (a) pristine, (b) EG3, and (c) EG20 films.

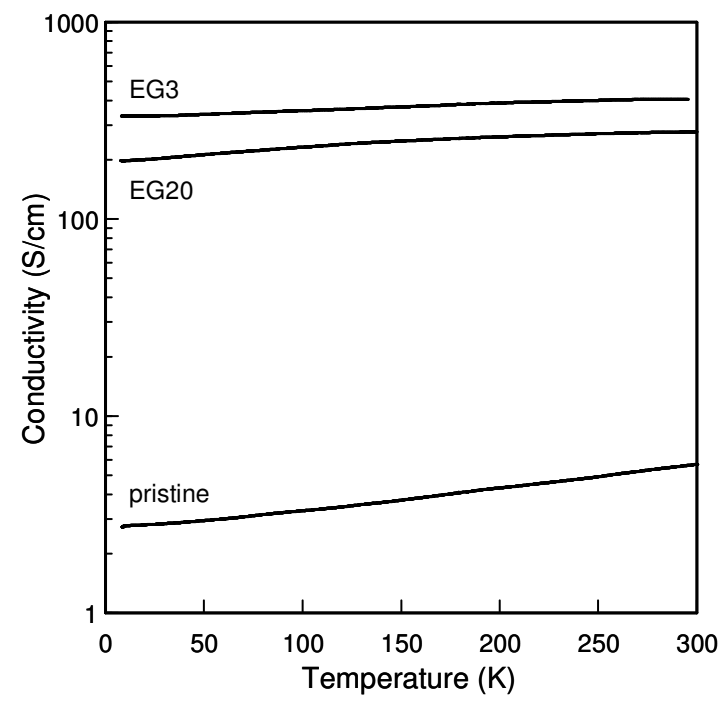

Fig.6 Temperature dependence of electrical conductivity of pristine, EG3, and EG20 films measured by a four-probe method from 8 to $300 \mathrm{~K}$ at a constant heating rate of $1 \mathrm{~K} / \mathrm{min}$.

According to a nearest-neighbor hopping model, activation energy $\left(E_{\mathrm{a}}\right)$ for the pristine film was calculated to be $18 \mathrm{meV}$ similar to that of microfibers [5], indicating the carrier transport 
properties is intrinsic to the pristine PEDOT/PSS regardless of the shape and size [23,24]. It is noted that the value of $E_{\mathrm{a}}$ significantly decreased to 2.7 meV for the EG3 film. The result reveals that intraand inter-particle transport of charge carriers is enhanced by addition of EG, suggesting that the large numbers of highly conductive domains form conductive paths for hopping the charge carriers in the film as indicated in Fig.5(b). However, the $E_{\text {a }}$ for the EG20 film slightly increases to $2.9 \mathrm{meV}$ though the highly conductive domains increase in size and in number as shown in Fig.5(c). This is ascribed to the morphological inhomogeneity due to the phase segregation that may interrupt transport of charge carriers between the highly conductive domains.

\section{CONCLUSIONS}

In conclusion, we succeeded in fabricating PEDOT/PSS films with the electrical conductivity as high as $430 \mathrm{~S} / \mathrm{cm}$ by addition of EG. The results of XPS, XRD, and AFM allowed us to conclude that the EG was crucially important for (i) removal of the insulating PSS from the surface of the PEDOT/PSS particles, (ii) crystallization of PEDOT molecules, and (iii) aggregation of the particles, which improved both intra- and inter-particle transfer of charge carriers. A further increase of the EG concentration, however, decreased the conductivity, which was due to the morphological inhomogeneity by the phase segregation.

\section{ACKNOWLEDGMENTS}

This work was supported in part by Grant for Industrial Technology Research Grant Program in 2008 and Practical Application of University R\&D Results under the Matching Fund Method from New Energy and Industrial Technology Development Organization (NEDO), Japan and Grant-in-Aid for Scientific Research from the Ministry of Education, Science, Sports, and Culture of Japan.

\section{REFERENCES}

[1] D. Hohnholz, A. G. MacDiarmid, H. Okuzaki, Adv. Funct. Mater., 15, 51-56 (2005).

[2] H. Yan, T. Jo, H. Okuzaki, Polym. J., 41, 1028-29 (2009).

[3] D. Hohnholz, A. G. MacDiarmid, Synth. Met., 121, 1327-28 (2001).

[4] H. Okuzaki, M. Ishihara, Macromol. Rapid Commun., 24, 261-64 (2003).

[5] H. Okuzaki, Y. Harashina, H. Yan, Euro. Polym. J., 45, 256-61 (2009).

[6] T. Takahashi, M. Ishihara, H. Okuzaki, Synth. Met., 152, 73-76 (2005).

[7] H. Yan, Y. Endo, Y. Hara, H. Okuzaki, Chem. Lett., 36, 986-87 (2007).

[8] H. Okuzaki, M. Ishihara, S. Ashizawa, Synth. Met., 137, 947-48 (2003).

[9] S. Ashizawa, Y. Shinohara, H. Shindo, Y. Watanabe, H. Okuzaki, Synth. Met., 153, 41-44 (2005).
[10] H. Okuzaki, S. Ashizawa, Multifunctional Conducting Molecular Materials, RSC Pub., (2007), pp.270-275.

[11] H. Okuzaki, H. Suzuki, T. Ito, J. Phys. Chem. $B$, 113, 11378-83 (2009).

[12] H. Okuzaki, K. Hosaka, H. Suzuki, T. Ito, Sens. Actuat. A, 157, 96-99 (2010).

[13] H. Okuzaki, H. Suzuki, T. Ito, Synth. Met., 159, 2233-36 (2009).

[14] S. Ashizawa, R. Horikawa, H. Okuzaki, Synth. Met., 153, 5-8 (2005).

[15] J. Y. Kim, J. H. Jung, D. E. Lee, J. Joo, Synth. Met., 126, 311-16 (2002).

[16] S. K. M. Jönsson, J. Birgerson, X. Crispin, G. Greczynski, W. Osikowicz, A. W. Denier van der Gon, W. R. Salaneck, M. Fahlman, Synth. Met.,139, 1-10 (2003).

[17] S. Ghosh and O. Inganäs, Synth. Met., 121, 1321-22 (2001).

[18] G. Greczynski, T. Kugler, W. R. Salaneck, Thin Solid Films, 354, 129-35 (1999).

[19] H. Yan, H. Okuzaki, Synth. Met., 159, 2225-28 (2009).

[20] G. Greczynski, T. Kugler, M. Keil, W. Osikowicz, M. Fahlman, W. R. Salaneck, J. Electr. Spectr. Rel. Phenom., 121, 1-17 (2001).

[21] K. E. Aasmundtveit, E. J. Samuelsen, L. A. A. Pettersson, O. Inganäs, T. Johansson, R. Feidenhans'1, Synth. Met., 101, 561-64 (1999).

[22] X. Crispin, F. L. E. Jakobsson, A. Crispin, P. C. M. Grim, P. Andersson, A. Volodin, C. van Haesendonck, M. Van der Auweraer, W. R. Salaneck, M. Berggren, Chem. Mater., 18, 4354-60 (2006).

[23] H. Yan, S. Arima, Y. Mori, T. Kagata, H. Sato, H. Okuzaki, Thin Solid Films, 517, 3299-303 (2009).

[24] H. Yan, H. Okuzaki, Macromol. Symp., 296, 286-93 (2010).

(Received Janurary 19, 2011; Accepted March 20, 2011) 